\title{
Quantum Hall Ferroelectrics and Nematics in Multivalley Systems
}

\author{
Inti Sodemann, ${ }^{1,2}$ Zheng Zhu, ${ }^{1}$ and Liang $\mathrm{Fu}^{1}$ \\ ${ }^{1}$ Department of Physics, Massachusetts Institute of Technology, Cambridge, Massachusetts 02139, USA \\ ${ }^{2}$ Max-Planck Institute for the Physics of Complex Systems, D-01187 Dresden, Germany \\ (Received 14 June 2017; revised manuscript received 1 October 2017; published 22 December 2017) \\ We study broken symmetry states at integer Landau-level fillings in multivalley quantum Hall systems \\ whose low-energy dispersions are anisotropic. When the Fermi surface of individual pockets lacks twofold \\ rotational symmetry, like in bismuth (111) [Feldman et al. , Observation of a Nematic Quantum Hall Liquid \\ on the Surface of Bismuth, Science 354, 316 (2016)] and in $\mathrm{Sn}_{1-x} \mathrm{~Pb}_{x} \mathrm{Se}$ (001) [Dziawa et al., Topological \\ Crystalline Insulator States in $\mathrm{Pb}_{1-x} \mathrm{Sn}_{x} \mathrm{Se}$, Nat. Mater. 11, 1023 (2012)] surfaces, interactions tend to drive \\ the formation of quantum Hall ferroelectric states. We demonstrate that the dipole moment in these states \\ has an intimate relation to the Fermi surface geometry of the parent metal. In quantum Hall nematic states, \\ like those arising in AlAs quantum wells, we demonstrate the existence of unusually robust Skyrmion \\ quasiparticles.
}

DOI: 10.1103/PhysRevX.7.041068

\section{INTRODUCTION}

Quantum Hall liquids with nearly degenerate internal degrees of freedom have long been the source of a rich variety of phenomena. Aside from multilayer systems, there exist today an array of multivalley two-dimensional electron systems exhibiting the quantum Hall effect, including quantum wells of AlAs [1], monolayer and bilayer graphene [2,3], silicon surfaces [4], PbTe (111) surfaces [5], surface states of the topological crystalline insulator $\mathrm{Sn}_{1-x} \mathrm{~Pb}_{x} \mathrm{Se}$ [6], and, more recently, the (111) surface of bismuth [7]. These systems all have multiple valleys related to each other by discrete crystal symmetries.

Among these multivalley systems, AlAs heterostructures, Si surfaces, PbTe (111) quantum wells, and $\mathrm{Bi}(111)$ surfaces all have highly anisotropic pockets, whose orientations are valley dependent. The shape of the Fermi surface is crucial in determining the pattern of valley symmetry breaking. For AlAs and $\mathrm{PbTe}$, the pockets are centered at time-reversal-invariant momenta and therefore possess an elliptical shape with twofold symmetry. This Fermi surface anisotropy favors valley-polarized states where a subset of valley-degenerate Landau levels (LL) are fully occupied $[8,9]$. This state spontaneously breaks the larger crystal rotational symmetry down to twofold rotations, and therefore, the resulting state is a nematic quantum Hall state.

However, the recently studied $\mathrm{Bi}(111)$ surface and $\mathrm{Sn}_{1-x} \mathrm{~Pb}_{x} \mathrm{Se}(001)$ surface, brings a novel ingredient into

Published by the American Physical Society under the terms of the Creative Commons Attribution 4.0 International license. Further distribution of this work must maintain attribution to the author(s) and the published article's title, journal citation, and DOI.
Subject Areas: Condensed Matter Physics

this problem. As shown in (Fig. 1), the Bi (111) surface has six tadpole-shaped hole pockets [10,11]; the $\mathrm{Sn}_{1-x} \mathrm{~Pb}_{x} \mathrm{Se}$ (001) surface has four crescent-shaped pockets [12]. These pockets come in time-reversed pairs located at opposite momenta away from time-reversal-invariant points in the Brillouin zone. Importantly, the Fermi surface of each pocket does not have a twofold rotational symmetry. Therefore, we generally expect Landau orbitals associated with each valley to carry an in-plane dipolar component. This implies that a valley-polarized state will be an insulator that spontaneously breaks inversion symmetry. We refer to this phase as a quantum Hall ferroelectric state. The giant Fermi surface anisotropy of $\mathrm{Bi}(111)$ and $\mathrm{Sn}_{1-x} \mathrm{~Pb}_{x} \mathrm{Se}(001)$ surface states makes them promising candidate systems to study this novel ferroelectricity in quantum Hall states (Fig. 1).

In this paper, we provide a unified description of these ferroelectric and nematic states in multivalley integer quantum Hall systems. We establish that, because of the Fermi surface anisotropy, the long-range Coulomb interaction generically favors states with full valley polarization. We compute the electric polarization of quantum Hall ferroelectrics using a quantum-mechanical approach based
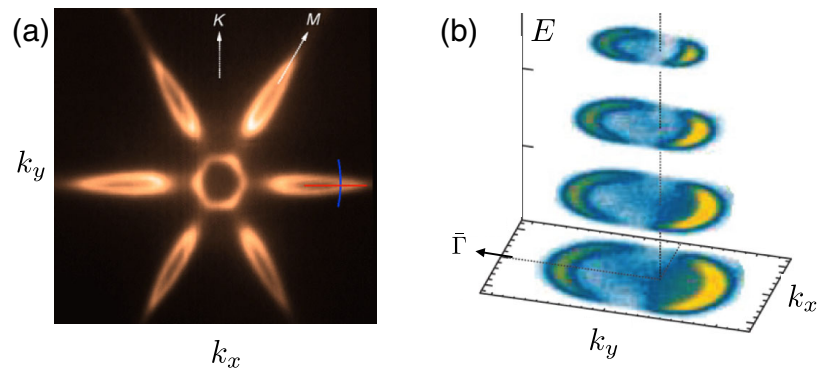

FIG. 1. ARPES Fermi surfaces of (a) the Bi(111) surface from Ref. [11] and (b) the $\mathrm{Sn}_{1-x} \mathrm{~Pb}_{x} \mathrm{Se}$ (001) surface from Ref. [12]. 
on the Berry phase and also using a semiclassical approach that directly relates the dipole moment to the underlying Fermi surface geometry. For quantum Hall nematic states, we study the energetics of Skyrmion-type charged excitations [13] using the density-matrix renormalization group (DMRG) method, and we find that they are surprisingly robust against mass anisotropy of the valleys, shedding new light on the experiments on AlAs quantum wells [14].

\section{GENERAL SETUP}

We start from symmetry considerations on twodimensional (2D) multivalley systems. We consider systems with threefold, fourfold, or sixfold rotational symmetry, which rules out any principal axis within the $2 \mathrm{D}$ plane. In order for each valley to have an anisotropic dispersion, we require that the symmetry group that leaves each valley invariant, or the "little group," is only a subgroup of $C_{2 v}$ or, equivalently, contains no more than a twofold rotation $(x, y) \rightarrow(-x,-y)$ and at most two mirror planes that are orthogonal to each other, $(x, y) \rightarrow(-x, y)$ and $(x, y) \rightarrow(x,-y)$.

Within this wide class of systems, it is convenient to distinguish two types of two-dimensional systems with multiple anisotropic valleys: Type I are those whose little group contains at most a single mirror plane; type II are those with larger symmetries. For type-I systems, each valley is of such low symmetry that the electron dispersion at zero field has no inversion center, i.e., $\epsilon(\mathbf{k}) \neq \epsilon(-\mathbf{k})$, where $\mathbf{k}$ is the "small" momentum within the valley. As we will show, in the quantum Hall regime and at odd-integer fillings, Coulomb interaction tends to induce a valleypolarized state that breaks all rotational symmetry of the crystal and is therefore a ferroelectric. For type-II systems, the electron dispersions at each valley have twofold symmetry $\epsilon(\mathbf{k})=\epsilon(-\mathbf{k})$, and the resulting valley-polarized state will retain $\pi$ rotations. In this case, valley-polarized quantum Hall states exhibit nematic instead of ferroelectric orders. The $\mathrm{Bi}(111)$ surface [7] is a representative of type-I systems, and the AlAs quantum well [1] is a representative of type-II systems.

To study interacting electrons in multivalley quantum Hall systems, we assume that the magnetic field is large enough so that the Hamiltonian can be projected into a set of $M$ degenerate Landau levels associated with different valleys. The system is at some integer filling $\nu \in \mathbb{Z}$ $(\nu \leq M)$. The interaction between an electron with position $\mathbf{r}_{1}$ from valley $i$ and an electron with position $\mathbf{r}_{2}$ from valley $j$ is dominated by the long-range part of the Coulomb interaction [15]:

$$
v\left(\mathbf{r}_{1}-\mathbf{r}_{2}\right)=\frac{e^{2}}{\epsilon\left|\mathbf{r}_{1}-\mathbf{r}_{2}\right|} .
$$

Despite the bare Coulomb interaction being valley independent, it effectively becomes valley dependent after projection into the Landau levels of interest:
$P_{L L} v\left(\mathbf{r}_{1}-\mathbf{r}_{2}\right) P_{L L}=\frac{1}{A} \sum_{\mathbf{q}} v_{\mathbf{q}} F_{I}(\mathbf{q}) F_{J}(-\mathbf{q}) e^{i \mathbf{q} \cdot\left(\mathbf{R}_{1}-\mathbf{R}_{2}\right)}$,

where $\mathbf{R} \equiv \mathbf{r}-l^{2} \hat{\mathbf{z}} \times \mathbf{p}$ is the intra-Landau-level guiding center operator, $\mathbf{p}=\boldsymbol{\nabla} / i-e \mathbf{A}$ is the mechanical momentum, and $F_{J}(\mathbf{q}) \equiv\left\langle J\left|e^{-i l^{2} \mathbf{\mathbf { z }} \cdot \mathbf{q} \times \mathbf{p}}\right| J\right\rangle$ is the form factor determined by the wave function, $|J\rangle$, of the Landau level of interest associated with valley $J\left(\hbar \equiv 1, l^{2} \equiv 1 / e B\right)$. These form factors are crucial for the energetics of valley symmetry-breaking quantum Hall states and are described for specific cases of type-I and type-II multivalley systems in Appendixes A-C.

We note that our approach rests on the assumption that the long-range Coulomb force projected into a set of valleydegenerate Landau levels is the dominant term that is able to break the many-body degeneracy and select the ground states. This assumption is justified in systems with anisotropic Fermi surfaces (which are the subject of this work), provided that short-range interactions are small. For systems with nearly isotropic Fermi surfaces such as graphene, the long-range Coulomb interaction leaves large remnant continuous symmetries; hence, short-distance corrections are needed to select the ground state (see, e.g., Refs. $[16,17]$ ). Additionally, for massless Dirac fermion systems, the Landau-level projection is only perturbatively enforced by the smallness of the effective fine-structure constant; hence, corrections beyond naive degenerate perturbation theory that include the role of the negative energy sea might be important when such a parameter is not small.

\section{HARTREE-FOCK THEORY}

We study the Hamiltonian (2) using both the HartreeFock approximation and the DMRG numerical method. Within Hartree-Fock, we consider translationally invariant trial Slater determinant states that are arbitrary coherent superpositions of the $M$ valleys. These states can be parametrized as follows:

$$
|\Psi\rangle=\prod_{a=1}^{\nu} \prod_{k=1}^{N_{\phi}}\left(\sum_{I=1}^{M}\left\langle\chi_{a} \mid I\right\rangle c_{k I}^{\dagger}\right)|\mathrm{O}\rangle
$$

where $|\mathrm{O}\rangle$ is the reference vacuum in which the Landau levels of interest are empty, $k$ is an intra-Landau-level guiding center label, $I$ labels the $M$ valleys, and $\left|\chi_{a}\right\rangle$ are $N$ orthonormal vectors describing the occupied coherent combinations of such valleys. The expectation value of the energy (with the Hartree part removed by including a neutralizing background) is

$$
\begin{aligned}
E[P] & =-N_{\phi} \sum_{I, J} X_{I J}\langle I|P| J\rangle\langle J|P| I\rangle, \quad P=\sum_{a=1}^{\nu}\left|\chi_{a}\right\rangle\left\langle\chi_{a}\right|, \\
X_{I J} & =\frac{1}{2} \int \frac{d^{2} \mathbf{q}}{(2 \pi)^{2}} v_{\mathbf{q}} F_{I}(\mathbf{q}) F_{J}^{*}(\mathbf{q}),
\end{aligned}
$$


where $I$ labels the valleys and $\left|\chi_{a}\right\rangle$ are $\nu$ orthonormal vectors describing the occupied coherent combinations of the valleys. Trial states are found by minimizing $E[P]$ as a function of $P$.

It is well known that, generically, at integer fillings, the ground states of multicomponent systems with repulsive interactions are spontaneous integer quantum Hall states with coherence between different components known as quantum Hall ferromagnets. We have found a generalized and stronger version of quantum Hall ferromagnetism at $\nu=1$ for the kind of multicomponent systems with anisotropic Fermi surfaces that we are considering. In particular, we show that the state that minimizes the Hartree-Fock energy is maximally polarized into a single valley, and those states with coherence between different valleys are energetically disfavored by the exchange energy. In fact, we prove the following theorem:

Full valley polarization theorem.-If the set of form factors $F_{I}(\mathbf{q}), I=\{1, \ldots, \nu\}$, are linearly independent functions of $\mathbf{q}$ and the interaction is strictly repulsive, $v_{\mathbf{q}}>0$ for all $\mathbf{q}$, the minimum of Hartree-Fock energy $E[P]$ at $\nu=1$ for any multivalley system is a state maximally polarized into a single valley.

Proof.-We begin by noting that, provided the interactions are repulsive, $v_{\mathbf{q}}>0$, the exchange integral defines an inner product for form factors as functions of $\mathbf{q}$ :

$$
\left\langle F_{I}, F_{J}\right\rangle \equiv X_{I J}=\frac{1}{2} \int \frac{d^{2} \mathbf{q}}{(2 \pi)^{2}} v_{\mathbf{q}} F_{I}(\mathbf{q}) F_{J}^{*}(\mathbf{q})
$$

Here, $X_{I J}$ is a real symmetric matrix whose entries are the inner products, known in linear algebra as a Gram matrix. A classic theorem establishes that if the vectors used in the Gram matrix are linearly independent, the matrix is strictly positive definite; namely, all its eigenvalues are real and positive. Therefore, we conclude that the linear independence of $F_{I}(\mathbf{q})$ implies that $X_{I J}$ is a strictly positive matrix.

On the other hand, at $\nu=1$, the expression for the exchange energy reduces to

$$
E=-N_{\phi} \sum_{I J} p_{I} X_{I J} p_{J}, \quad p_{I}=|\langle I \mid \chi\rangle|^{2},
$$

where $|\chi\rangle$ is the single trial coherent combination of the valleys that is occupied at $\nu=1$. The task is to find the minimum of this energy as a function of probabilities $p_{I}$ that define a point in $\mathbb{R}^{M}$ that is further restricted to a region defined by the constraints $\sum_{I} p_{I}=1, p_{I} \in[0,1]$. Let us denote this region by $\mathbf{P}$, which is illustrated in Fig. 2. Now, since $X_{I J}$ is strictly positive definite, it defines itself as an inner product in $\mathbb{R}^{M}$ :

$$
\mathbf{p}_{1} \cdot \mathbf{p}_{2} \equiv \sum_{I J} p_{1 I} X_{I J} p_{2 J}
$$

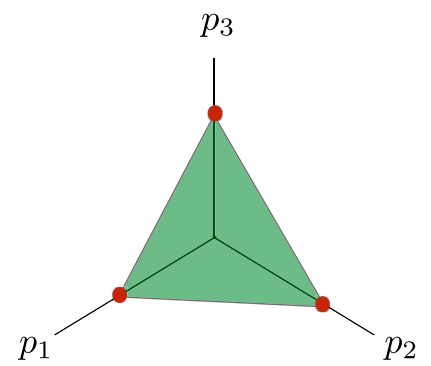

FIG. 2. The green triangular region defines the allowed values of $p_{I}$, and the red dots at the corners represent the fully valleypolarized states in the case of $M=3$.

Now, consider a line in $\mathbb{R}^{M}$ parametrized by $t \in[0,1]$, $\mathbf{p}(t)=t \mathbf{p}_{1}+(1-t) \mathbf{p}_{2}$, connecting two distinct points, $\mathbf{p}_{1} \neq \mathbf{p}_{2}$. It is easy to show that if $\mathbf{p}_{1} \in \mathbf{P}$ and $\mathbf{p}_{2} \in \mathbf{P}$, then $\mathbf{p}(t) \in \mathbf{P}, \forall t \in[0,1]$. Notice that

$$
\begin{aligned}
& \|\mathbf{p}(t)\|^{2} \equiv \mathbf{p}(t) \cdot \mathbf{p}(t) \\
& \quad=\left\|\mathbf{p}_{1}-\mathbf{p}_{2}\right\|^{2} t^{2}+2 t\left(\mathbf{p}_{1} \cdot \mathbf{p}_{2}-\left\|\mathbf{p}_{2}\right\|^{2}\right)+\left\|\mathbf{p}_{2}\right\|^{2}
\end{aligned}
$$

Since the coefficient of $t^{2}$ is strictly positive, then the maximum of $\|\mathbf{p}(t)\|^{2}$ is always achieved at the end points of the line and never strictly within its interior. Since the energy is $E=-N_{\phi}\|\mathbf{p}\|^{2}$, it follows that any point that can be viewed as being strictly in the interior of a line contained in $\mathbf{P}$ cannot be a minimum of the energy. Therefore, the minima of the energy must necessarily be located at the corners of $\mathbf{P}$ because these are the only points that cannot be viewed as being strictly in the interior of a line contained within $\mathbf{P}$. The $J$ th corner is defined by $p_{J}=1$ and $p_{I}=0$ for $I \neq J$. The state with the minimum energy is therefore fully valley polarized, and the valley with minimum energy will be the one with the largest exchange integral $X_{J J}$. In the case in which the valleys are related by a discrete crystal symmetry, the exchange integral $X_{J J}$ will be the same for every $J$, and we get a discrete degeneracy of all the fully polarized states. Thus, we conclude our proof that the system spontaneously fully polarizes into a single valley.

A simple way to test that form factors are linearly independent is to check that $\operatorname{det}\left(X_{I J}\right) \neq 0$. It is easy to imagine that form factors that arise from valleys with anisotropic Fermi surfaces are generically linearly independent. This behavior is the generic case for valleys with anisotropic Fermi surfaces that are distinct. We use the word distinct here in the strong sense that the Fermi surfaces are not identical upon translation in momentum space. For example, two elliptical Fermi surfaces that are rotated with respect to each other, as in AlAs, are distinct. Similarly, all the Fermi surfaces at the Bi(111) surface are distinct (Fig. 1). Therefore, we expect, generically, that the ground states of type-I and type-II systems are fully valleypolarized ferroelectric or nematic states at $\nu=1$. 
It is possible, however, that two or more Fermi surfaces for distinct valleys are, in a small momentum approximation, identical (as in the case of graphene within the leading linear $k$ "dot" $p$ Dirac approximation); hence, the form factors are linearly dependent. These systems, in the presence of long-range Coulomb interaction, have an emergent $\mathrm{SU}(N)$ symmetry between the $N$ valleys with identical form factors. Also, an important condition for the preceding analysis to apply is that all electrons reside in the same layer so that the interactions before projecting to the Landau level are identical. Otherwise, nontrivial Hartree terms may favor superpositions of components in different layers, as it happens for the exciton condensate in quantum Hall bilayers [18].

In fact, the fully valley-polarized states are exact eigenstates of the many-body Hamiltonian projected into the Landau levels of interest. This follows from the constraint of valley number conservation in our model, which implies that, after projection into the multivalley Landau level of interest, they have no other states in the Hilbert space to mix with. It is more subtle to demonstrate that they are the lowest-energy states. As we have seen, however, this is the expectation based on Hartree-Fock theory. We have confirmed via density-matrix renormalization group numerical simulations on the torus geometry [19-23] that they are indeed the exact ground states, as discussed in Sec. VII.

Although the valley-polarization theorem predicts fully valley-polarized states in a general class of systems, one should be mindful of potential subtleties in specific systems that would require going beyond the simple model we have described. One consideration is the importance of shortdistance corrections to the Coulomb interaction, which typically are reduced from the energy scale of the Coulomb interaction by a factor of the order of $a / l$, where $a$ is a lattice length scale. A simple way to decide when these terms are important is to compare their size with the energy difference per particle between a valley-polarized state and a valley-coherent state within the description we have provided. When such a difference is small, the tendency to select a unique ground state by using the Coulomb interaction alone would be weak. In such cases, one should consider the role of these short interactions in selecting ground states on an equal footing with the asymmetry of the form factors we have described, in an analogous fashion to how it has been done in graphene [16].

\section{QUANTUM HALL FERROELECTRIC DIPOLE MOMENT}

Following our full valley-polarization theorem, we expect type-I systems to exhibit valley-polarized states at filling $\nu=1$, which fully breaks rotational symmetry. The presence of a macroscopic dipole moment is a subtle issue. Unlike conventional ferroelectrics, quantum Hall ferroelectrics are always accompanied by a chiral metallic edge. Therefore, one expects that any polarization charge that accumulates at the boundary of the sample will be screened by the metallic edge [24]. In the forthcoming discussion, we ignore this subtlety in the measurement of the dipole moment. In Sec. VI A, we discuss how the dipole moment that we compute can be experimentally measured from the charge distribution of quasiparticles.

\section{A. General expressions for the dipole moment}

The dipole of the insulating state at $\nu=1$ can be defined as the change of the average position of the electrons relative to a reference state with inversion symmetry. Since the state at $\nu=1$ is a Slater determinant, the dipole moment per electron can be computed for each single orbital as a single-particle property. We illustrate two different ways of computing the dipole moment, which result in identical final results but are useful as they illustrate different points of view on the quantum-mechanical theory of polarization.

The first approach starts from the expression that the dipole moment associated with an orbital $k$ in a valley $J$ is given by the change of the expectation value of the position operator:

$$
\mathbf{D}=-|e|\left(\langle k, J|\mathbf{r}| k, J\rangle-\langle k, J|\mathbf{r}| k, J\rangle_{0}\right) .
$$

Here, $k$ is an intra-Landau level guiding center label, and $J$ labels the valley that is spontaneously chosen in the ground state. For purposes of defining the dipole moment, we consider a hypothetical reference Hamiltonian that has inversion symmetry, whose eigenfunctions are $|k, J\rangle_{0}$, and we imagine that this Hamiltonian can be adiabatically deformed into the Hamiltonian of interest describing the dispersion of valley $J$, which breaks inversion symmetry. The dipole is obtained as the change in the position relative to the reference inversion-symmetric state. The position operator can be decomposed into cyclotron and guiding center components as follows:

$$
\mathbf{r} \equiv \mathbf{R}-\hat{\mathbf{z}} \times \mathbf{p},
$$

where $\mathbf{p}=\nabla / i-\mathbf{A}(\mathbf{r})$ is the gauge-invariant mechanical momentum. The key observation is that the guiding center variables are adiabatic invariants because guiding center operators commute with the mechanical momentum, $\left[R_{a}, p_{b}\right]=0$, and the Hamiltonian describing each valley is a function of $\mathbf{p}$ throughout the entire adiabatic path because the path is assumed not to break translational symmetry. Thus, by choosing the origin of coordinates to be the initial position of the orbital, so that $\langle k, J|\mathbf{r}| k, J\rangle_{0}=0$, we arrive at the following expression for the dipole moment:

$$
\mathbf{D}=-|e| l^{2} \hat{\mathbf{z}} \times\langle J|\mathbf{p}| J\rangle .
$$

Here, we have omitted explicit reference to the guiding center label of the eigenstate in question as the formula 
makes manifest that the dipole moment is independent from it. The formula just derived offers great versatility for computations of the dipole moment for specific systems when the cyclotron eigenstates are known explicitly in terms of the canonical cyclotron raising and lowering operators because $\mathbf{p}$ is a simple linear function of these operators. We take advantage of this to perform a swift computation of the dipole moment in tilted Dirac cones, which are relevant for the surface of topological crystalline insulators described in Sec. V.

The second approach follows from adapting the formalism of the modern theory of polarization $[25,26]$ to our current problem. To compute the dipole moment along a given direction within this approach, we choose a gauge so that there is translational invariance along such a direction. For example, to compute the dipole along $y$, we choose $A_{x}=0$ and $A_{y}=B x$. In this case, the single-particle states within the Landau level of interest can be labeled by a momentum $k_{y}$ and have a real-space form:

$$
\psi_{k_{y}}(x, y)=\frac{e^{i k_{y} y}}{\sqrt{L_{y}}} u_{k_{y}}(x)
$$

Periodicity along the $y$ direction imposes a discretization of $k_{y}\left(\Delta k_{y}=2 \pi / L_{y}\right)$, and the finite size and the periodicity of the system along the $x$ direction determine the size of the effective Brillouin zone to be $k_{y} \in\left[0, L_{x} / l^{2}\right]$. To determine the polarization, we compute the integral over time of the current operator:

$$
I_{y}=-|e| \frac{\partial H_{k_{y}}}{\partial k_{y}}
$$

in response to an adiabatically changing Dirac cone tilt $\delta v_{x}(t)$ over a period of time $T$, so that $\delta v_{x}(t=0)=0$ and $\delta v_{x}(t=T)=\delta v_{x}$. Following the arguments of Refs. $[25,26]$, one finds the following expression for the change in the dipole moment per particle:

$$
D_{y}=-i|e| \frac{l^{2}}{L_{x}} \int_{0}^{L_{x} / l^{2}} d k_{y}\left\langle u_{k_{y}}\left|\partial_{k_{y}}\right| u_{k_{y}}\right\rangle
$$

In the present case, the real-space wave functions at different $k_{y}$ differ by a translation: $u_{k_{y}}(x)=u_{0}\left(x-k_{y} l^{2}\right)$. As a consequence, the formula reduces to

$$
\begin{aligned}
D_{y} & =-\left.i|e|\left\langle u_{k_{y}}\left|\partial_{k_{y}}\right| u_{k_{y}}\right\rangle\right|_{k_{y}=0} \\
& =i|e| l^{2} \int d x u_{0}^{*}(x) \partial_{x} u_{0}(x) .
\end{aligned}
$$

To show the equivalence of the two approaches to compute the dipole moment, we recast Eq. (15) as follows:

$$
\begin{aligned}
D_{y} & =i|e| l^{2} \int d^{2} r \psi_{k_{y}}^{*}(x, y) \partial_{x} \psi_{k_{y}}(x, y)=|e| l^{2}\left\langle\psi_{k_{y}}\left|p_{x}\right| \psi_{k_{y}}\right\rangle \\
& =-|e|\left\langle\psi_{k_{y}}\left|\left(y-R_{y}\right)\right| \psi_{k_{y}}\right\rangle .
\end{aligned}
$$

The last expression shows that the dipole moment computed in this way coincides with the average position of the single-particle orbital measured with respect to the guiding center $R_{y}$.

\section{B. WKB approximation for the dipole moment}

We now introduce a semiclassical approach to establish a direct connection of the dipole moment with the underlying Fermi surface geometry at zero field. Consider a valley described by a single-band Hamiltonian $H\left(p_{x}, p_{y}\right)$ of arbitrary form. For simplicity, we imagine that the eigenstates near this valley have negligible Berry phase or, equivalently, that the Hamiltonian has only differential operators but no pseudospin matrix structure. This is typically a good approximation near the bottom of a band for a single-band system provided other bands are sufficiently far in energy. As in the previous subsection, by choosing the Landau gauge $A_{x}=0$ and $A_{y}=B x$, we view the eigenvalue problem as effectively one dimensional. The eigenvalue problem reads as

$$
H\left(\frac{\hbar}{i} \frac{d}{d x}, k_{y}-e B x\right) u_{k_{y}}(x)=\epsilon_{n} u_{k_{y}}(x) .
$$

Without loss of generality, we can set $k_{y}=0$ since other solutions are obtained by a global translation. We can search for an approximate WKB solution to this differential equation, which formally is an expansion in $\hbar$ :

$$
u_{0}(x)=\exp \left(\frac{i}{\hbar} \phi_{0}(x)+\phi_{1}(x)+\mathcal{O}(\hbar)\right) .
$$

Substituting this expression into Eq. (17) and keeping corrections up to linear order in $\hbar$ leads to the WKB approximation of the problem [27]:

$u_{0}(x) \approx \sum_{s} \frac{1}{\sqrt{v_{x, s}^{\mathrm{cl}}(x, \epsilon)}} \exp \left(\frac{i}{\hbar} \int^{x} d x^{\prime} p_{x, s}^{\mathrm{cl}}\left(x^{\prime}, \epsilon\right)\right)$,

where $p_{x, s}^{\mathrm{cl}}\left(x^{\prime}, \epsilon\right)$ is the $s$ root of the algebraic equation defined by the classical dispersion relation: $H\left(p_{x, s}^{\mathrm{cl}},-e B x\right)=\epsilon$, and

$$
v_{x, S}^{\mathrm{cl}}(x, \epsilon)=\left.\frac{\partial H\left(p_{x},-e B x\right)}{\partial p_{x}}\right|_{p_{x} \rightarrow p_{x, s}^{\mathrm{cl}}(x, \epsilon)}
$$

is the group velocity evaluated on the classical trajectory. Typically, we have two roots $s=+,-$ and two turning points $x_{+,-}$that separate the classically allowed from the 
classically forbidden regions. In the classically forbidden regions, $p_{x, s}^{\mathrm{cl}}$ is imaginary and the wave function decays exponentially. As is well known, the quantization condition for the WKB solution is obtained by matching boundary conditions between classically allowed and forbidden regions, and it leads to the Onsager quantization condition [28], which, in the absence of the Berry phase, reduces to

$\sum_{s} \frac{s}{e B} \int_{p_{y}^{-}}^{p_{y}^{+}} d p_{y} p_{x, s}^{\mathrm{cl}}\left(p_{y}, \epsilon\right)=l^{2} A\left(\epsilon_{n}\right)=2 \pi(n+1 / 2)$,

where we made the change of variables $p_{y}=-e B x, A\left(\epsilon_{n}\right)$ is the area in momentum space inside the iso-energetic contour $H\left(k_{x}, k_{y}\right)=\epsilon_{n}$, and $n$ is the Landau-level index. From Eq. (15), we write the dipole as $D_{y}=$ $-e l^{2} \int d x \operatorname{Im}\left(u_{0}^{*}(x) \partial_{x} u_{0}(x)\right)$. There are two types of contributions to the dipole moment, namely, the sum of the contributions coming from each classical root separately and the contribution corresponding to the interference-like crossed terms between the two classical roots. The semiclassical approximation is expected to work when the oscillations of the wave function occur over a length much smaller than that in which the confining potential changes; therefore, we restrict ourselves to states that have a high energy and hence a large Landau-level index. For these states, the crossed terms have rapid oscillatory factors of the form $\exp \left(i / \hbar \int^{x} d x^{\prime}\left(p_{x, 1}^{\mathrm{cl}}-p_{x, 2}^{\mathrm{cl}}\right)\right)$ and can be neglected. In addition, in this case, the integrals can be approximated to be taken over the classically allowed region because the states with rapid oscillations in the classically allowed region correspondingly have fast decay in the classically forbidden region. Therefore, the terms in which the oscillatory part cancels within the classically allowed region have the dominant contribution. As a consequence, we obtain

$$
\begin{aligned}
\int & d x \operatorname{Im}\left(u_{0}^{*}(x) \partial_{x} u_{0}(x)\right) \\
& \approx-\sum_{s} \frac{s}{e B} \int_{p_{y}^{-}}^{p_{y}^{+}} d p_{y} \frac{1}{\left|v_{x, s}^{\mathrm{cl}}\left(p_{y}, \epsilon\right)\right|} p_{x, s}^{\mathrm{cl}}\left(p_{y}, \epsilon\right) \\
& =-\oint d t p_{x}(t, \epsilon),
\end{aligned}
$$

where, in the last line, we have established the connection to the classical cyclotron motion described in the previous section, in which $d \mathbf{r}(t) / d t=\mathbf{v}=l^{2} d \mathbf{k}(t) / d t \times \hat{z}$, from which we have that $v_{x}=l^{2} d k_{y} / d t$. Additionally, we must normalize the WKB solution. Using the same approximations employed to compute the dipole, one obtains that the approximate normalization of the WKB solution is

$$
\int d x u^{*}(x) u(x) \approx \sum_{s} \frac{1}{e B} \int_{p_{y}^{-}}^{p_{y}^{+}} d p_{y} \frac{1}{\left|v_{x, s}^{\mathrm{cl}}\left(p_{y}, \epsilon\right)\right|}=\oint d t .
$$

Thus, the normalization is simply the total time that it takes to complete the classical cyclotron orbit. A similar analysis can be followed to find the dipole along the $x$ direction, $D_{x}$. Therefore, within the approximations outlined here, the WKB quantized wave function predicts that the dipole moment is simply given by the timeaveraged position of the electron over the semiclassical orbit:

$$
\mathbf{D} \approx-|e|\left\langle\mathbf{r}\left(t, \epsilon_{n}\right)\right\rangle_{t} \equiv-|e| \frac{\oint d t \mathbf{r}\left(t, \epsilon_{n}\right)}{\oint d t},
$$

where the integral is performed over the semiclassical cyclotron orbit $\mathbf{r}(t)=l^{2} \mathbf{k}(t) \times \hat{z}$, with $\mathbf{k}(t)$ tracing a constant energy contour $H(\mathbf{k}(t))=\epsilon_{n}$ at a speed $v=|\partial \epsilon / \partial \mathbf{k}|$ [29]. This picture intuitively and generically predicts that the dipole is orthogonal to the direction of the distortion of the Fermi surface since the real-space orbit is a rotated version of the Fermi surface.

\section{QUANTUM HALL FERROELECTRICS AT THE SURFACE OF TOPOLOGICAL CRYSTALLINE INSULATORS}

There are various material platforms for type-I multivalley systems, where anisotropic valleys are located at non-time-reversal-invariant momenta and each valley lacks twofold symmetry. One candidate platform is the multivalley system on the (111) surface of bismuth [10,11]. Another interesting platform, on which we focus in this section, is the (001) surface of topological crystalline insulators (TCI) $\mathrm{SnTe}, \mathrm{Sn}_{x} \mathrm{~Pb}_{1-x} \mathrm{Se}$, and $\mathrm{Sn}_{x} \mathrm{~Pb}_{1-x} \mathrm{Te}$ [30]. In both $\mathrm{Bi}(111)$ and TCI's, surface-state Landau levels have been observed by means of scanning tunneling microscopy $[6,7]$.

To study ferroelectricity at the surface of TCI's, we consider a model with two valleys $(M=2)$ of Dirac fermions located at opposite momenta, at filling $\nu=1$. This model describes the low-energy dispersion of the [001] surface of SnTe and PbSnTe topological crystalline insulators in the low-temperature phase [31]. Here, the Dirac cones are generically "tilted" [32] and described by the following Hamiltonian:

$$
H_{0}= \pm v_{x} \sigma_{x} p_{x}+v_{y} \sigma_{y} p_{x} \pm \delta v_{x} p_{x} .
$$

The only discrete symmetry that leaves each valley invariant is a single mirror plane along the line that connects the Dirac cones [31,32]. We consider the $n=1$ Landau level [33]. The wave functions associated with each valley break rotation and inversion symmetry as illustrated in Fig. 3. Using the form factors described in Appendix $\mathrm{C}$ and applying Eq. (4) to the case of $M=1$ at $\nu=1$, the Hartree-Fock energy can be found as follows (up to a global constant): 

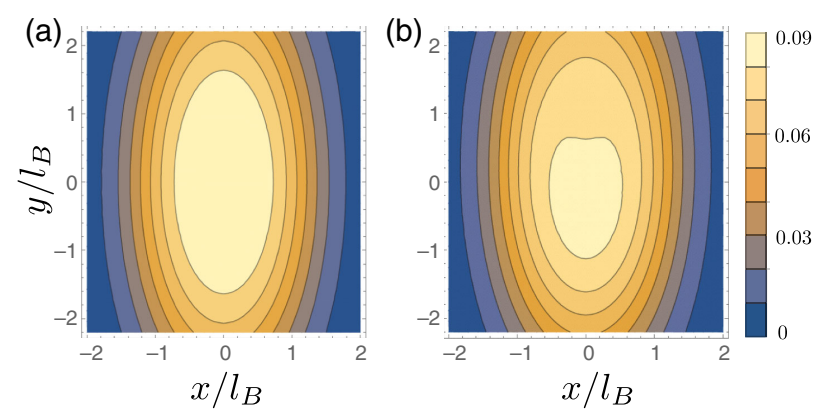

FIG. 3. Probability amplitude contours for coherent states in the first Landau level of a Dirac cone with anisotropic velocities $v_{x} / v_{y} \approx 2.2$ (a) and with anisotropy and tilt $\delta v_{x} \approx 0.3$ (b). The wave function carries a dipole moment perpendicular to the tilt of the Dirac cone.

$$
E / A=-\beta n_{z}^{2},
$$

where $n_{z}=\operatorname{tr}\left(\sigma_{z} P\right)$, with $\sigma_{z}$ a Pauli matrix in the valley indices, and $A$ is the system area. Here, $\beta$ is a positive constant, in agreement with the theorem of full valley polarization, and it is explicitly given by

$$
\beta=\frac{\tau^{2}}{4 \pi} \int \frac{d^{2} q}{(2 \pi)^{2}} v_{q} e^{-|\bar{q}|^{2} / 2}\left(3-\frac{|\bar{q}|^{2}}{2}\right)^{2} \bar{q}_{y}^{2}
$$

where $\tau=\delta v_{x} /\left(\sqrt{2} v_{y}\right) \quad$ and $\quad\left(\bar{q}_{x}, \bar{q}_{y}\right)=\left(\sqrt{v_{y} / v_{x}} q_{y}\right.$, $\left.\sqrt{v_{x} / v_{y}} q_{x}\right)$. Therefore, the ground state is an Ising-type $\left(n_{z}= \pm 1\right)$ ferroelectric. The dipole moment, to leading order in the Dirac cone tilt, can be computed using the form of the Landau levels in terms of cyclotron raising and lowering operators (Appendix C), and using the Eq. (11); it is found to be

$$
D_{y} \approx \pm|e l| \operatorname{sign}(B) \frac{3 \delta v_{x}}{\sqrt{2} v_{y}}, \quad D_{x}=0 .
$$

The dipole of each valley is orthogonal to the direction of the Dirac cone tilt and reverses with the direction of the perpendicular field. This is allowed since the magnetic field breaks the mirror symmetry present at zero field. In our discussion, we have neglected the Zeeman coupling. This approximation is justified when the Zeeman energy is much smaller than the cyclotron spacing of the Dirac Landau levels in question, but including the Zeeman term should not qualitatively change the results in these systems.

\section{EXPERIMENTAL MANIFESTATIONS OF QUANTUM HALL FERROELECTRICS}

We now discuss potential experimental manifestations of the quantum Hall ferroelectrics. In the case of the $\mathrm{Bi}(111)$ surface [7], we expect these states to appear at odd-integer fillings. The degree of broken inversion asymmetry in this system is expected to increase with the Landau-level index because the lowest Landau levels might be well described by an elliptical dispersion near the bottom of the hole bands, for which no dipole moment is expected but only nematic states reported experimentally [7]. Because this system has coexisting ferroelectric and nematic character, one signature is the appearance of additional ferroelectric domain walls in addition to those associated with nematicity seen at even fillings. One interesting possibility is the existence of gapless edge states at the ferroelectric domain walls. If one neglects intervalley scattering, the valley pseudospin is conserved, and one expects gapless charge-carrying counterpropagating edge modes that arise at the domain walls. Because of their ferroelectric nature, these domains may be manipulated by STM bias voltage or in-plane external electric field. Finally, we note that Bi (111) hole pockets located at opposite momenta carry opposite in-plane spin polarizations, which cancel when the two are equally occupied. Therefore, valley-polarized states at odd-integer filling also carry an in-plane spin polarization that could be manipulated with in-plane magnetic fields. However, the broken inversion symmetry of this state would manifest directly in the charge distribution of quasiparticles, which would carry a dipole moment given by the formulas described in Sec. IV. We note in passing that other types of quantum Hall ferroelectrics arising in wide quantum wells [34] and in bilayer graphene [35] have been previously proposed, but to our knowledge, these proposals have not been experimentally realized so far.

\section{A. Measurement and physical meaning of the dipole moment}

The dipole moment we have computed is defined as the change of the average position of every electron in a Landau level along a path that connects the Hamiltonian of interest with a reference initial Hamiltonian that has inversion symmetry. The argument presented in Sec. IV A demonstrates that, as long as there is translational invariance, such a change is independent of the guiding center variables; hence, it is the same for any orbital constructed within a given Landau level.

As we noted in Sec. IV, the presence of the metallic edge of quantum Hall states prevents the buildup of charge at the boundaries of quantum Hall ferroelectrics. This may invalidate conventional approaches to measure the polarization used in ordinary ferroelectrics that rely on the measurement of macroscopic charge accumulation at the boundaries. Experimental manifestations of the formation of this inversion-symmetry-breaking quantum Hall state therefore require looking at other kinds of observables. One instance of the manifestation of the broken inversion symmetry is the charge distribution of quasiparticles. One consequence of inversion symmetry is that the charge distribution of elementary quasiparticles will be inversion symmetric. However, this will no longer be true when the 
system breaks inversion symmetry. Consider the elementary quasihole of the system, which is created by removing the electron from a single orbital $\psi_{k, J}(\mathbf{r})$, with an intraLandau label $k$, in the Slater determinant associated with completely filling a Landau level that we label by $J$. The particle density in the quasihole state is

$$
\rho_{q h}(\mathbf{r})=\frac{1}{2 \pi l^{2}}-\left|\psi_{k, J}(\mathbf{r})\right|^{2},
$$

where $1 / 2 \pi l^{2}$ is the background density of the $\nu=1$ state. Now, from this equation, we can see that the change of the average position of the quasihole state computed along an adiabatic path that connects the Hamiltonian of interest with a reference inversion-symmetric Hamiltonian will coincide with the change of position of the single-particle state $\psi_{k, J}(\mathbf{r})$ with an overall opposite sign. Therefore, the change of the dipole moment of the quasihole is exactly minus the dipole moment that we have described in Sec. IV.

Broadly speaking, the quasihole can be considered as a limiting version of a hole inside the sample that separates the quantum Hall ferroelectric and vacuum. When the size of this hole is very large, there are gapless excitations that accompany the formation of the chiral edge, which will screen the buildup of charge. However, when the size of the hole is small or comparable to the magnetic length, these excitations will acquire a finite-size gap, and we expect that dipole moments will build up in such a limit. Therefore, a dipole moment should build up for cavities or holes inside the sample that are on the order of the magnetic length, resembling the cavity electric fields that are used as a classic method in elementary discussions of dielectrics as a way to measure the electric dipole moment [36]. Strictly speaking, a cavity is different from a free quasihole because there is an explicit potential that expels electrons from its interior and breaks translational symmetry, which was one of our assumptions on the derivation of the dipole moment. Studying in detail the dipole moment buildup in such cavities is an interesting open question that we hope will be addressed in future work.

\section{QUANTUM HALL NEMATICS}

In this section, we consider type-II multivalley systems that develop nematicity but carry no dipole moment. We focus on the case with two valleys whose anisotropic mass tensors are rotated by a $\pi / 2$ angle relative to each other, as realized in AlAs $[1,8]$. Our main objective is to study nontrivial charged excitations, but we briefly review ground-state properties. At filling $\nu=1$, one finds that the energy is (up to a global constant) [8]

$$
E / A=-\alpha n_{z}^{2},
$$

where $n_{z}=\operatorname{tr}\left(\sigma_{z} P\right)$ and $\alpha>0$. The system has Ising character, in agreement with the theorem of full valley polarization. To further assess the validity of the HartreeFock approach, we have performed DMRG simulations, which are described in Appendix D. It is in fact easy to convince oneself that the Ising nematic state is an exact eigenstate of the Hamiltonian projected into the valleydegenerate Landau level. We confirmed that it is the ground state via DMRG employing a torus with a square aspect ratio (Appendix D) for as many as 24 electrons and for mass anisotropies as large as $m_{x} / m_{y}=16$.

\section{A. Valley Skyrmions in quantum Hall nematics}

Having established that the ground state is a nematic state, we now study its charged excitations. In the SU(2) invariant limit, i.e., when the mass tensors are identical for both valleys, we expect the lowest-energy excitation to be infinite-sized Skyrmions [13]. When the mass tensors are slightly different, we expect that the Skyrmions will have a finite size dictated by the competition between the Ising anisotropy, which wants to shrink them, and the Coulomb energy, which wants to expand them to smear the charge over large distances [37]. To be able to accurately study the properties of Skyrmions, we resort to DMRG [38]. The Skyrmion quasielectron can be obtained as the ground state of the Hamiltonian when $N=N_{\phi}+1$ [40]. Figure 4 shows that nontrivial Skyrmions (those involving at least one spin flip) survive up to large mass ratio $m_{x} / m_{y} \approx 3.8$. AlAs has mass anisotropy of about $m_{x} / m_{y} \approx 5$ [1]. The experiment of Ref. [14] found a nonlinear dependence of the charge gap on strain, which was interpreted as evidence for Skyrmions. Our findings suggest that this scenario is not unlikely since the critical mass ratio to observe Skyrmions is ultimately dependent on the details of interactions and can easily change by effects beyond our model (e.g., finite well widths and Landau-level mixing).

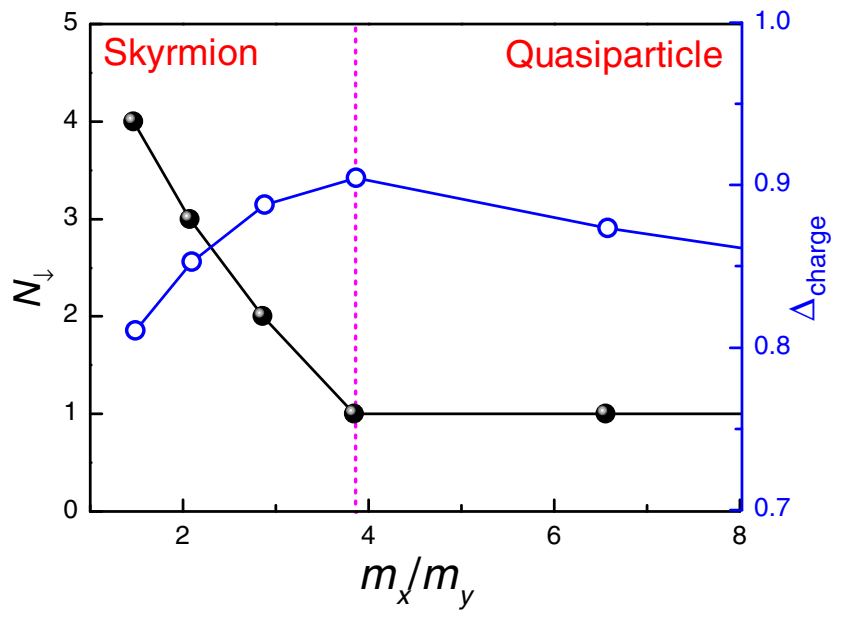

FIG. 4. Number of minority valley electrons (left axis) for quasiparticles as a function of mass anisotropy in a two-valley system (AlAs) and their charge gap (right axis). The charge gap is in Coulomb energy units of $e^{2} /(\epsilon l)$. 
We have also computed the charge gaps for these quasiparticles in Fig. 4. The charge gap of a system can be defined as the jump in chemical potential. The chemical potential is the energy change for adding a particle. Thus, the chemical potential to add a quasielectron on top of the ground state is $\mu_{+}=E_{N=N_{\phi}+1}-E_{N=N_{\phi}}$; similarly, the chemical potential for quasiholes is $\mu_{-}=E_{N=N_{\phi}}-E_{N=N_{\phi}-1}$. With these results, the charge gap is found to be

$$
\Delta_{\text {charge }}=\mu_{+}-\mu_{-}=E_{N=N_{\phi}+1}+E_{N=N_{\phi}-1}-2 E_{N=N_{\phi}} .
$$

A detailed comparison of the charge gaps of the nontrivial Skyrmions we have found with those expected within Hartree-Fock theory is presented in Appendix E. Finally, although we focused on the Skyrmions in the nematic case, Skyrmions could also be present in the ferroelectric states. These Skyrmions also carry a dipole moment, although its magnitude cannot be inferred from the simple formulas we have developed. The precise pattern of the Skyrmion texture in this case might be very complex because of the interplay of breaking of inversion symmetry and the long-range nature of Coulomb interactions. We hope future work addresses the nature of these interesting quasiparticles.

\section{SUMMARY}

In summary, we have shown that multivalley systems with anisotropic dispersions generically lead to ferroelectric or nematic quantum Hall states at odd-integer Landaulevel fillings. The ferroelectric states arise when the parent Fermi surface of a single valley lacks an inversion center, such as in the case of $\mathrm{Bi}(111)$ [7]. We have shown that the resulting dipole moment has an intimate relation with the underlying Fermi surface geometry of the parent metal. We also demonstrated the existence of nontrivial charged Skyrmion excitations in the nematic states with an unexpectedly large stability to the Ising symmetry-breaking terms, shedding light on the question of the presence of these excitations in AlAs [14].

\section{ACKNOWLEDGMENTS}

We would like to thank Vidya Madhavan, Benjamin Feldman, Malika Randeria, Ali Yazdani, Donna Sheng, Heun Mo Yoo, Joonho Jang, Ray Ashoori, Fengcheng Wu, and Allan H. MacDonald for stimulating discussions. We especially thank Pok Man Tam for discussions and for catching a mistake in the numerical prefactor of Eqs. (28) and (C3) in an earlier unpublished version of the manuscript. Z.Z. is supported by the David and Lucile Packard Foundation. L. F. is supported by the DOE Office of Basic Energy Sciences, Division of Materials Sciences and Engineering under Grant No. DE-SC0010526.

\section{APPENDIX A: LANDAU LEVELS AND DENSITY FORM FACTORS FOR ANISOTROPIC PARABOLIC DISPERSIONS}

Consider the problem of Landau levels with an anisotropic mass tensor:

$$
H=\frac{1}{2 m^{*}} p_{a} g_{a b} p_{b}
$$

where $p=\nabla / i-e A$, and $g$ is a tensor that can be diagonalized as $g=Q^{T} S^{2} Q$, where $Q \in S O(2)$ and $S$ is diagonal with positive eigenvalues and $\operatorname{det} S=1$. Explicitly, $S=\operatorname{diag}\left\{\left(m_{x} / m_{y}\right)^{1 / 4},\left(m_{y} / m_{x}\right)^{1 / 4}\right\}$ and $m^{*}=$ $\left(m_{x} m_{y}\right)^{1 / 2}$. We can define rescaled momenta along the principal axes of the tensor as $\pi_{a}=(S Q)_{a b} p_{b}$; these satisfy the same commutation relations as the original ones:

$$
\left[\pi_{a}, \pi_{b}\right]=i l^{-2} \epsilon_{a b},
$$

which allows us to solve the LL problem by defining the LL raising operators:

$$
a \equiv \frac{l}{\sqrt{2}}\left(\pi_{x}+i \pi_{y}\right)
$$

satisfying $\left[a, a^{\dagger}\right]=1$. The guiding center operators are intra-Landau-level operators defined as

$$
R_{a} \equiv r_{a}+l^{2} \epsilon_{a b} p_{b}
$$

They satisfy

$$
\begin{aligned}
& {\left[R_{a}, R_{b}\right]=-i l^{2} \epsilon_{a b},} \\
& {\left[R_{a}, p_{b}\right]=\left[R_{a}, \pi_{b}\right]=0 .}
\end{aligned}
$$

The single-particle Hilbert space can be decomposed into a tensor product $|n\rangle \otimes|m\rangle$, where $|n\rangle$ are the Landaulevel indices on which $a, a^{\dagger}$ act and $|m\rangle$ are intra-Landaulevel indices on which $R_{a}$ acts. Now, the projected interaction into the Landau level of interest is obtained by imagining we have two flavors of particles with different mass tensors $\left(g_{1}=Q_{1}^{T} S_{1}^{2} Q_{1}\right.$ and $\left.g_{2}=Q_{2}^{T} S_{2}^{2} Q_{2}\right)$ that interact via a potential that depends only on interparticle distance:

$$
V\left(r_{1}-r_{2}\right)=\frac{1}{A} \sum_{q} V_{q} e^{i q \cdot\left(r_{1}-r_{2}\right)}
$$

where $r_{1}$ and $r_{2}$ above are understood to be operators. Using Eq. (A4), we decompose the position of each particle as

$$
r_{1} \equiv R_{1}-l^{2} \epsilon p_{1}=R_{1}-l^{2} \epsilon Q_{1}^{T} S_{1}^{-1} \pi_{1},
$$


where we use matrix notation for the two-component LeviCivita symbol $\epsilon$. We have a similar expression for $r_{2}$. Using this expression, we get

$$
\begin{aligned}
e^{i q \cdot\left(r_{1}-r_{2}\right)} & =e^{i q \cdot\left(R_{1}-R_{2}\right)} e^{-i l^{2}\left(q_{1} \cdot \pi_{1}-q_{2} \cdot \pi_{2}\right)}, \\
q_{i} & =-S_{i}^{-1} Q_{i} \epsilon q .
\end{aligned}
$$

In the above expression, the terms containing the operator $\pi_{1}$ produce inter-Landau-level mixing, and we proceed by projecting them into the zeroth Landau level, which is defined as $a|0\rangle=0$. By using Eq. (A3) in combination with the $\mathrm{BCH}$ formula, one can show that

$$
e^{i l^{2} q \cdot \pi}=e^{-\left[\left(l^{2}|q|^{2}\right) / 4\right]} e^{[(i l) / \sqrt{2}] \mathbf{q} a^{\dagger}} e^{[(i l) / \sqrt{2}] \mathbf{q}^{*} a},
$$

where $\mathbf{q}=q_{x}+i q_{y}$. Then, one obtains that the projected Hamiltonian is

$P_{0} V\left(r_{1}-r_{2}\right) P_{0}=\frac{1}{A} \sum_{q} V_{q} e^{-l^{2}\left[\left(\left|q_{1}\right|^{2}+\left|q_{2}\right|^{2}\right) / 4\right]} e^{i q \cdot\left(R_{1}-R_{2}\right)}$.

Notice that $q_{1,2}$ are linear functions of $q$ described in Eq. (A8).

For higher Landau levels, we need to modify the density form factors. Using Eq. (A9) and the algebra of raising and lowering operators, one can show the following identities:

$$
\begin{aligned}
& \left\langle n\left|e^{i l^{2} q \cdot \pi}\right| n\right\rangle \\
& =e^{-\left[\left(l^{2}|q|^{2}\right) / 4\right]} \sum_{m=0}^{n}\left(-\frac{l^{2}|q|^{2}}{2}\right)^{m} \frac{n(n-1) \ldots(n-m+1)}{(m !)^{2}}, \\
& =e^{-\frac{l^{2}|q|^{2}}{4}} L_{n}\left(\frac{l^{2}|q|^{2}}{2}\right)
\end{aligned}
$$

where $n$ is the Landau level of interest and $L_{n}$ are the Laguerre polynomials. Therefore, the interaction projected to the $n$ Landau level is

$$
\begin{aligned}
& P_{n} v\left(r_{1}-r_{2}\right) P_{n} \\
& =\frac{1}{A} \sum_{q} v_{q} L_{n}\left(\frac{l^{2}\left|q_{1}\right|^{2}}{2}\right) L_{n}\left(\frac{l^{2}\left|q_{2}\right|^{2}}{2}\right) \times e^{-l^{2} \frac{\left|q_{1}\right|^{2}+\left|q_{2}\right|^{2}}{4}} e^{i q \cdot\left(R_{1}-R_{2}\right) .}
\end{aligned}
$$

\section{APPENDIX B: LANDAU LEVELS AND DENSITY FORM FACTORS FOR ANISOTROPIC DIRAC CONES}

The derivation is very similar to that of Galilean electrons. We start from an anisotropic Dirac Hamiltonian:

$$
H=\sigma_{a} g_{a b} p_{b}=v \sigma_{a}^{\prime} \pi_{a},
$$

where $g$ is a $3 \times 2$ tensor to which we apply a singular-value decomposition of the form $g=R^{T} S Q$, where $R \in S O$ (3) describes a rotation of Pauli matrices $\sigma_{a}$ in pseudospin space, $Q \in S O(2)$ describes the transformation of the principal axes in real space, and $S$ is a $3 \times 2$ matrix whose upper $2 \times 2$ block is diagonal and characterizes the anisotropy of velocities $S=\operatorname{diag}\left\{\left(v_{x} / v_{y}\right)^{1 / 2},\left(v_{y} / v_{x}\right)^{1 / 2}\right\}$, and whose lower row has zero entries, and $v=\left(v_{x} v_{y}\right)^{1 / 2}$. Using the same definition of ladder operators as in Eq. (A3), the Hamiltonian and the spectrum are

$$
\begin{aligned}
H & =\frac{\sqrt{2} v}{l}\left(\begin{array}{cc}
0 & a^{\dagger} \\
a & 0
\end{array}\right), \\
|n, k, s\rangle_{D} & =\frac{1}{\sqrt{2}}\left(\begin{array}{c}
|n, k\rangle \\
s|n-1, k\rangle
\end{array}\right), \quad \text { for } n>0 \\
|0, k\rangle_{D} & =\left(\begin{array}{c}
|0, k\rangle \\
0
\end{array}\right), \quad E_{n k s}=s \frac{\sqrt{2} n v}{l},
\end{aligned}
$$

where $s= \pm$ labels negative- and positive-energy Landau levels and $k$ is the guiding center intra-Landau-level index. From these expressions, we can obtain the form factors of the interaction projected to the Dirac Landau levels. One can show that the zero Landau level is identical to the Galilean case, and for the excited Landau levels, one gets the same expressions discussed in the previous section but with the modification

$L_{n}\left(\frac{l^{2}|q|^{2}}{2}\right) \rightarrow \frac{1}{2}\left(L_{n}\left(\frac{l^{2}|q|^{2}}{2}\right)+L_{n-1}\left(\frac{l^{2}|q|^{2}}{2}\right)\right)$.

\section{APPENDIX C: LANDAU LEVELS AND DENSITY FORM FACTORS IN TILTED DIRAC CONES}

To the Hamiltonian $H$ appearing in Eqs. (B1) and (B2), we add a perturbation describing the tilt of the Dirac cone along the $x$ direction:

$$
H_{1}=\delta v_{x} \pi_{x}=\delta v_{x} \sqrt{\frac{v_{x}}{v_{y}}} \frac{\left(a+a^{\dagger}\right)}{\sqrt{2} l},
$$

where $a$ is defined in Eq. (A3). The (un-normalized) perturbed zero-energy Landau level to first order in $\delta v_{x}$ is found to be

$$
|0\rangle_{1}=\left(\begin{array}{c}
|0\rangle \\
-\tau|0\rangle
\end{array}\right)
$$

where $\tau=\delta v_{x} /\left(\sqrt{2} v_{y}\right)$. This verifies that the zero Landau level does not carry a dipole as expected since particle-hole times inversion is a symmetry. Generically, the $n$th Landau level will, however, break inversion symmetry. The valence and conduction first Landau levels have a perturbed form: 


$$
| \pm 1\rangle_{1}=\frac{1}{\sqrt{2}}\left(\begin{array}{c}
|1\rangle \pm \tau(|0\rangle-2 \sqrt{2}|2\rangle) \\
\pm|0\rangle-3 \tau|1\rangle
\end{array}\right) .
$$

As described in the main text, we now imagine two Dirac valleys at opposite momenta and with tilts $\delta v_{x}$ of opposite sign and equal magnitude. Let us assume we are filling only one of the two first Landau levels. The form factor obtained from the above perturbative expression is

$$
\begin{aligned}
F_{\tau}(q) \equiv & { }_{1}\left\langle 1\left|e^{i l^{2} q \cdot \pi^{\prime}}\right| 1\right\rangle_{1} \approx \frac{1}{2}\left[f_{11}(q)+f_{00}(q)\right. \\
& \left.-2 \tau\left(f_{10}(q)+f_{01}(q)\right)-2 \sqrt{2} \tau\left(f_{21}(q)+f_{12}(q)\right)\right],
\end{aligned}
$$

where $f_{n m}(q) \equiv e^{-l^{2}|q|^{2} / 4}\left\langle n\left|e^{i l^{2} q \cdot \pi^{\prime}}\right| m\right\rangle$. To leading order in $\tau$, we can write the form factor as

$$
F_{\tau}(q) \approx e^{-l^{2}|q|^{2} / 4}\left[\left(1-\frac{q^{2}}{4}\right)-\sqrt{2} i \tau q_{y}\left(3-\frac{q^{2}}{2}\right)\right] .
$$

One valley will have a form factor corresponding to $\tau$ and the other corresponding to $-\tau$ since the tilts have opposite signs. We label them by $\alpha=\{+,-\}$. If one performs the calculation of the exchange energy with these form factors, then one finds the exchange integrals in Eq. (4),

$X_{\alpha \beta}=\frac{1}{2} \int \frac{d^{2} q}{(2 \pi)^{2}} v_{q} F_{\alpha}(\bar{q}) F_{\beta}(\bar{q}), \quad \bar{q}=-S^{-1} Q \epsilon q$.

The exchange energy can then be shown as

$$
E / N_{\phi}=\frac{\left(1-n_{z}^{2}\right)}{2} X_{\tau}+\text { const, }
$$

with

$$
\begin{aligned}
X_{\tau} & =\frac{X_{++}+X_{--}-X_{+-}-X_{-+}}{2} \\
& =\tau^{2} \int \frac{d^{2} q}{(2 \pi)^{2}} v_{q} e^{-|\bar{q}|^{2} / 2}\left(3-\frac{|\bar{q}|^{2}}{2}\right)^{2} \bar{q}_{y}^{2} .
\end{aligned}
$$

Clearly, for a repulsive interaction with $v_{q}>0$, we get that $X_{\tau}$ is strictly positive. Therefore, the ground state is the Ising nematic ferroelectric state: $n_{z}= \pm 1$.

\section{APPENDIX D: DMRG IMPLEMENTATION}

Numerically, we consider $N$ electrons moving along the surface of a torus with a magnetic field perpendicular to its surface. Note that $L_{x}\left(L_{y}\right)$ represents the circumference of the torus along the $x(y)$ direction and satisfies the relation $L_{x} L_{y}=2 \pi N_{\phi}$, where $N_{\phi}$ represents the number of orbitals. Here, we set the magnetic length $l_{B} \equiv \sqrt{\hbar c / e B}$ as the unit of length. We choose a square torus with aspect ratio 1 , i.e., $L_{x}=L_{y}$. Periodic boundary conditions require $k_{y} \equiv 2 \pi j / L_{y}$, with $j=0,1, \ldots, N_{\phi}-1$. The Coulomb interaction on finite-size system has the form

$$
V(\mathbf{r})=\frac{1}{L_{x} L_{y}} \sum_{\mathbf{q}} V(q) \exp (i \mathbf{q} r),
$$

with $V(q)=2 \pi e^{2} / L_{x} L_{y} \varepsilon q$, and the wave vectors are chosen to guarantee the periodicity of the interaction $V\left(x+L_{x}, y\right)=V\left(x, y+L_{y}\right)=V(x, y)$. The projected Coulomb interaction between particles $i, j$ into the lowest Landau level of a valley with mass anisotropic dispersion has the form

$$
V\left(\mathbf{R}_{i}-\mathbf{R}_{j}\right)=\sum_{\mathbf{q}} V(q) e^{-q_{1}^{2} / 2} e^{i \mathbf{q}\left(\mathbf{R}_{i}-\mathbf{R}_{j}\right)},
$$

where $q_{1}$ is a linear function of $q$ controlled by the anisotropic mass tensors described in Eq. (A8). The matrix elements take the explicit form

$$
\begin{aligned}
V_{j_{1} j_{2} j_{3} j_{4}}= & \delta^{\prime}{ }_{j_{1}+j_{2}, j_{3}+j_{4}} \frac{1}{2 L_{x} L_{y}} \sum_{\mathbf{q}, \mathbf{q} \neq 0} \delta^{\prime}{ }_{j_{1}-j_{4}, q_{y} L_{y} / 2 \pi} \\
& \times \frac{2 \pi e^{2}}{\varepsilon q} \exp \left[-q_{1}^{2} / 2-i\left(j_{1}-j_{3}\right) q_{x} L_{x} / N_{\phi}\right] .
\end{aligned}
$$

Here, the Kronecker delta with the prime means that the equation is defined modulo $N_{\phi}$. We also consider a uniform and positive background charge so that the Coulomb interaction at $q=0$ is absent [41].

For the case of two anisotropic valleys, the Coulomb interaction includes both the intracomponent and intercomponent interactions. After projecting onto the lowest Landau level, the effective Hamiltonian reads

$$
\begin{aligned}
V= & \sum_{i<j} \sum_{\mathbf{q}} V(q) e^{-q_{1}^{2} / 2} e^{i \mathbf{q}\left(\mathbf{R}_{1, i}-\mathbf{R}_{1, j}\right)} \\
& +\sum_{i<j} \sum_{\mathbf{q}} V(q) e^{-q_{2}^{2} / 2} e^{i \mathbf{q}\left(\mathbf{R}_{2, i}-\mathbf{R}_{2, j}\right)} \\
& +\sum_{i<j} \sum_{\mathbf{q}} V(q) e^{-\left(q_{1}^{2}+q_{2}^{2}\right) / 4} e^{i \mathbf{q}\left(\mathbf{R}_{1, i}-\mathbf{R}_{2, j}\right)} .
\end{aligned}
$$

Here, the first two terms are the intracomponent Coulomb interaction, and the last term is the intercomponent Coulomb interaction; $q_{1,2}$ are the linear functions of $q$ controlled by the anisotropic mass tensors of each valley described in Eq. (A8). An expression similar to Eq. (D3) holds for the explicit matrix elements.

Simulations are realized by mapping the single-particle orbitals into a one-dimensional lattice. The advantage of the DMRG method, compared to exact diagonalization, is that it can achieve reliable results for larger system sizes. In this paper, we impose the momentum-space DMRG method on 
a torus, where each site in the $x$ direction corresponds to different states labeled by the momentum in the $y$ direction. The standard procedure is similar to real-space DMRG, but since the nonzero Coulomb interaction exists between the orbitals satisfying the momentum conservation $j_{1}+j_{2}=$ $j_{3}+j_{4} \bmod N_{\phi}$, only a small number of momentum sectors in each DMRG block contribute to the ground state in the initial process. To ensure the convergence, one has to save more momentum sectors by keeping a small number of states in these sectors during the initial process, which may become important in the later DMRG sweep, while the remaining states are selected following the standard DMRG algorithm to reach the required truncation accuracy. For our calculation, we keep around 2000-8000 states and perform measurements during each DMRG sweep until the results converge.

We found that, in the ground state, the charge is uniformly distributed into the two orbitals for the isotropic case [42], i.e., $m_{x} / m_{y}=1$, while the ground state is realized in a sector in which all electrons polarize into a single orbital with the same flavor for the anisotropic case, as shown in Fig. 5 for the $N=N_{\phi}=24$ system with $\left(m_{x} / m_{y}\right)^{1 / 4}=1.5,2$.

To study charged excitations, we search for ground states with one particle added to those described above. The behavior of the charged excitations is markedly distinct for large and small anisotropic ratios $m_{x} / m_{y}$. When the anisotropic ratio is large enough, as shown in Fig. 6 for the $N=N_{\phi}+1=21$ system with $\left(m_{x} / m_{y}\right)^{1 / 4}=2, N_{\phi}$ electrons will fully occupy the orbitals with the same flavor, while the extra one will stay at the orbital with another flavor. The position of this extra electron is determined by the total momentum $K /\left(2 \pi / N_{\phi}\right)$ targeted in DMRG simulation, but different momentum sectors have the same

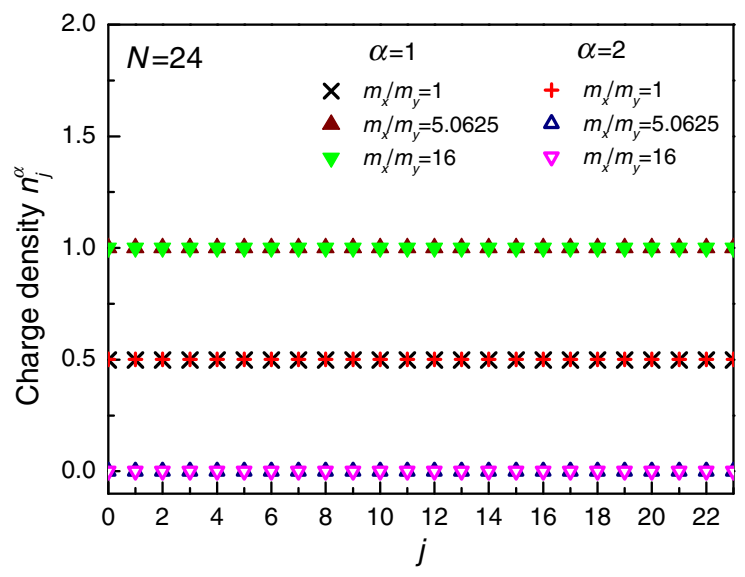

FIG. 5. The charge density distribution for the $N=24$ $\left(N_{\phi}=N\right)$ system with anisotropic ratio $m_{x} / m_{y}=1,5.0625$, 16. All of the electrons tend to stay in the same flavor $\alpha$ orbitals $\left(j=0,2, \ldots, N_{\phi}-1\right)$ for the anisotropic case. Here, $\alpha=1,2$ represents two components.

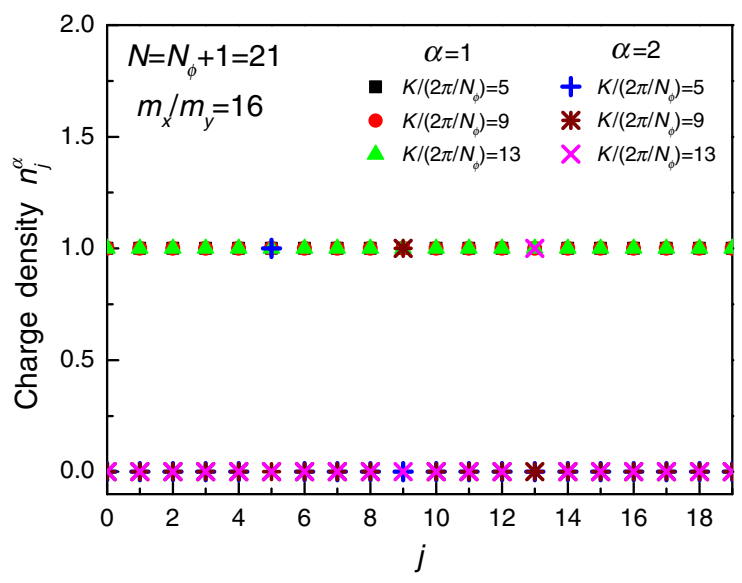

FIG. 6. The charge density distribution for the $N=21$ $\left(N_{\phi}=20\right)$ system with anisotropic ratio $m_{x} / m_{y}=16$. The extra electron will stay in the orbital $K /\left(2 \pi / N_{\phi}\right)$ if one targets the corresponding total momentum, and the ground-state energies are the same for different targeting momentum sectors. The total electron numbers for two flavors are $N_{1}=20$ and $N_{2}=1$.

energy because of translational invariance. This is consistent with the behavior of the conventional quasiparticle predicted by Hartree-Fock analysis. However, for smaller $m_{x} / m_{y}$ ratios, as shown in Fig. 7 with $\left(m_{x} / m_{y}\right)^{1 / 4}=1.2$, the total number of electrons in the majority flavor will be smaller than $N_{\phi}$, while the number of particles in the minority flavor will be larger than 1 , indicating that the quasiparticle has a nontrivial pseudospin texture. Here, the number of particles in each flavor depends on both anisotropic ratio $m_{x} / m_{y}$ and total number of particles $N$, and therefore, extrapolations to $N \rightarrow \infty$ are required. Figure 8 shows the finite-size scaling of the charge gap in

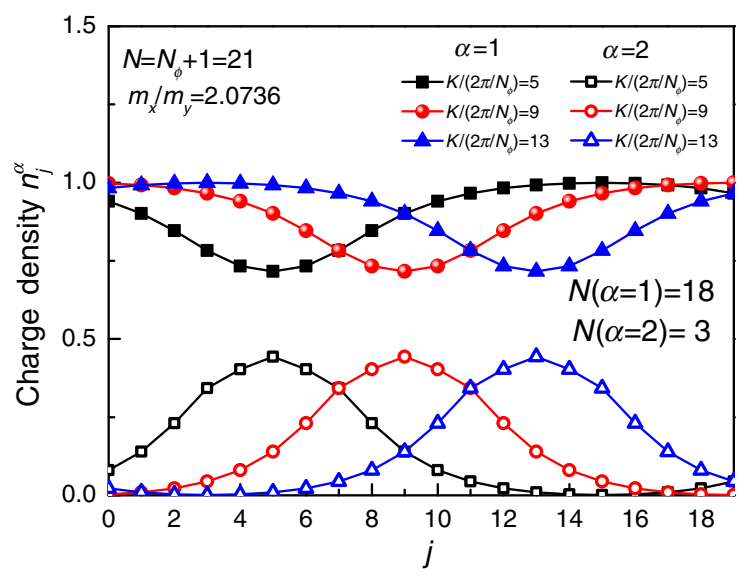

FIG. 7. The charge density distribution for the $N=21$ $\left(N_{\phi}=20\right)$ system with anisotropic ratio $m_{x} / m_{y}=2.0736$. The extra electron will stay near the orbital $K /\left(2 \pi / N_{\phi}\right)$ if one targets the corresponding total momentum, and the ground-state energies are the same for different targeting momentum sectors. The electron numbers for two flavors are $N_{1}=18$ and $N_{2}=3$, depending on the system size. 


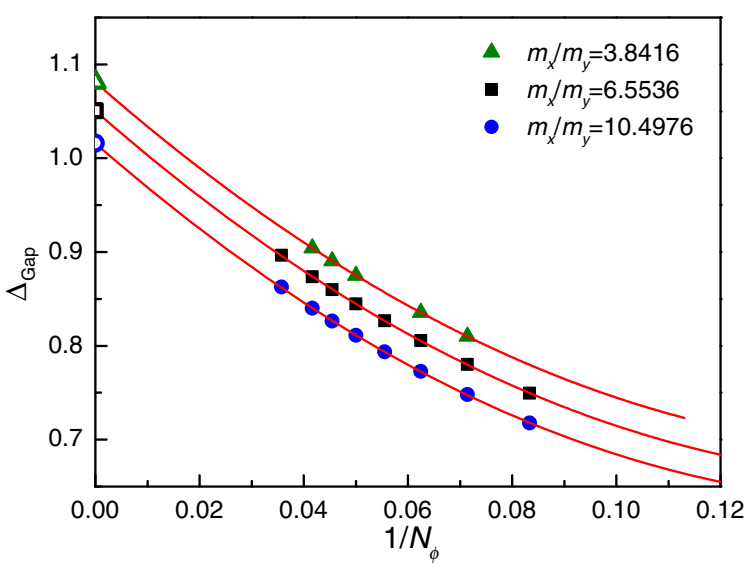

FIG. 8. The finite-size scaling of the charge gap in the quasiparticle regime for $m_{x} / m_{y}=6.5536$ and $m_{x} / m_{y}=10.4976$. The system size ranges from $N_{\phi}=12$ to $N_{\phi}=28$.

the quasiparticle regime. We calculate the charge gap $\Delta_{\text {gap }}$ for the systems with $N_{\phi}=12$ to $N_{\phi}=28$ and the anisotropic ratio $\left(m_{x} / m_{y}\right)^{1 / 4}=1.6$ and $\left(m_{x} / m_{y}\right)^{1 / 4}=1.8$. We fit the data as a quadratic function of $1 / N_{\phi}$ and estimate the thermodynamic limit of the gap by extrapolating $1 / N_{\phi} \rightarrow 0$.

\section{APPENDIX E: COMPARISON BETWEEN HARTREE-FOCK AND DMRG QUASIPARTICLE GAP}

The charge gap in Hartree-Fock theory can be shown to coincide with minus twice the energy per particle of the ground state, which, for the Coulomb interaction, can be found as

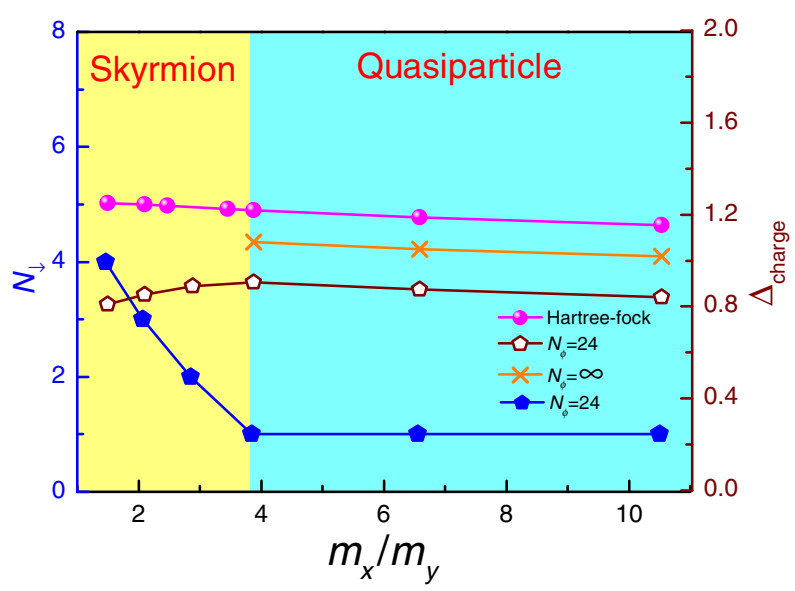

FIG. 9. The left vertical axis shows the number of spin flips (blue) involved in the lowest-energy charged quasiparticle as a function of mass anisotropy for a system with two anisotropic pockets like AlAs. The right vertical axis shows different calculations of the charge gap including Hartree-Fock (purple) and DMRG for the largest system size (white) and the extrapolated values to infinite size (yellow).

$$
\Delta_{\text {charge }}^{\mathrm{HF}}=-2 \frac{E_{\mathrm{HF}}^{0}}{N_{\phi}}=\frac{K\left(1-m_{y} / m_{x}\right)}{\left(m_{x} / m_{y}\right)^{1 / 4}} \sqrt{\frac{2}{\pi}} \frac{e^{2}}{l} .
$$

Here, $E_{\mathrm{HF}}^{0}$ is the Hartree-Fock energy of the ground state with no quasiparticles, and $K$ is the elliptic integral of the first kind. This formula corrects a typo in Ref. [8]. Figure 9 illustrates the Hartree-Fock charge gap for the conventional quasielectron and quasihole pair computed within Hartree-Fock theory and compared to the results from DMRG. After extrapolation to infinite sizes, the results show good agreement in the large-mass anisotropy regime where quasiparticles are no longer expected to be Skyrmions.

The physical reason for the decreasing gap at large anisotropies is that, as the anisotropy increases, the overlap between orbitals becomes smaller and their exchange energy gain is reduced. Because the energy gain per particle is reduced, it is also less energetically costly to add or remove particles, making the gap a decreasing function of mass anisotropy.

[1] M. Shayegan, E. P. De Poortere, O. Gunawan, Y. P. Shkolnikov, E. Tutuc, and K. Vakili, Two-Dimensional Electrons Occupying Multiple Valleys in AlAs, Phys. Status Solidi (b) 243, 3629 (2006).

[2] Y. Zhang, Y.-W. Tan, H. L. Stormer, and P. Kim, Experimental Observation of the Quantum Hall Effect and Berry's Phase in Graphene, Nature (London) 438, 201 (2005).

[3] K. S. Novoselov, E. McCann, S. V. Morozov, V. I. Fal'ko, M. I. Katsnelson, U. Zeitler, D. Jiang, F. Schedin, and A. K. Geim, Unconventional Quantum Hall Effect and Berry's Phase of $2 \pi$ in Bilayer Graphene, Nat. Phys. 2, 177 (2006).

[4] K. Eng, R. N. McFarland, and B. E. Kane, Integer Quantum Hall Effect on a Six-Valley Hydrogen-Passivated Silicon (111) Surface, Phys. Rev. Lett. 99, 016801 (2007).

[5] V. A. Chitta, W. Desrat, D. K. Maude, B. A. Piot, N. F. Oliveira Jr., P. H. O. Rappl, A. Y. Ueta, and E. Abramof, Integer Quantum Hall Effect in a PbTe Quantum Well, Physica E (Amsterdam) 34, 124 (2006).

[6] Y. Okada, M. Serbyn, H. Lin, D. Walkup, W. Zhou, C. Dhital, M. Neupane, S. Xu, Y. J. Wang, R. Sankar, F. Chou, A. Bansil, M.Z. Hasan, S. D. Wilson, L. Fu, and V. Madhavan, Observation of Dirac Node Formation and Mass Acquisition in a Topological Crystalline Insulator, Science 341, 1496 (2013).

[7] B. E. Feldman, M. T. Randeria, A. Gyenis, F. Wu, H. Ji, R. J. Cava, A. H. MacDonald, and A. Yazdani, Observation of a Nematic Quantum Hall Liquid on the Surface of Bismuth, Science 354, 316 (2016).

[8] D. A. Abanin, S. A. Parameswaran, S. A. Kivelson, and S. L. Sondhi, Nematic Valley Ordering in Quantum Hall Systems, Phys. Rev. B 82, 035428 (2010); A. Kumar, S. A. Parameswaran, and S. L. Sondhi, Microscopic Theory of a Quantum Hall Ising Nematic: Domain Walls and Disorder, Phys. Rev. B 88, 045133 (2013). 
[9] X. Li, F. Zhang, and A. H. MacDonald, SU(3) Quantum Hall Ferromagnetism in SnTe, Phys. Rev. Lett. 116, 026803 (2016).

[10] C. R. Ast and H. Höchst, Fermi Surface of Bi(111) Measured by Photoemission Spectroscopy, Phys. Rev. Lett. 87, 177602 (2001).

[11] Y. Ohtsubo, J. Mauchain, J. Faure, E. Papalazarou, M. Marsi, P. Le Fevre, F. Bertran, A. Taleb-Ibrahimi, and L. Perfetti, Giant Anisotropy of Spin-Orbit Splitting at the Bismuth Surface, Phys. Rev. Lett. 109, 226404 (2012).

[12] P. Dziawa, B. J. Kowalski, K. Dybko, R. Buczko, A. Szczerbakow, M. Szot, E. Lusakowska, T. Balasubramanian, B. M. Wojek, M. H. Berntsen, O. Tjernberg, and T. Story, Topological Crystalline Insulator States in $\mathrm{Pb}_{1-x} \mathrm{Sn}_{x} \mathrm{Se}$, Nat. Mater. 11, 1023 (2012).

[13] S. L. Sondhi, A. Karlhede, S. A. Kivelson, and E. H. Rezayi, Skyrmions and the Crossover from the Integer to Fractional Quantum Hall Effect at Small Zeeman Energies, Phys. Rev. B 47, 16419 (1993).

[14] Y. P. Shkolnikov, S. Misra, N. C. Bishop, E. P. De Poortere, and M. Shayegan, Observation of Quantum Hall "Valley Skyrmions”, Phys. Rev. Lett. 95, 066809 (2005).

[15] We neglect short-distance valley-dependent corrections. Note also that $v$ is independent of the relative orientation of the two electrons because systems considered here all have isotropic dielectric properties.

[16] M. Kharitonov, Phase Diagram for the $\nu=0$ Quantum Hall State in Monolayer Graphene, Phys. Rev. B 85, 155439 (2012); M. Kharitonov, Edge Excitations of the Canted Antiferromagnetic Phase of the $\nu=0$ Quantum Hall State in Graphene: A Simplified Analysis, Phys. Rev. B 86, 075450 (2012).

[17] G. Murthy, E. Shimshoni, and H. Fertig, Spin-Valley Coherent Phases of the $\nu=0$ Quantum Hall State in Bilayer Graphene, arXiv:1709.02447.

[18] K. Moon, H. Mori, K. Yang, S. M. Girvin, A. H. MacDonald, L. Zheng, D. Yoshioka, and S.-C. Zhang, Spontaneous Interlayer Coherence in Double-Layer Quantum Hall Systems: Charged Vortices and Kosterlitz-Thouless Phase Transitions, Phys. Rev. B 51, 5138 (1995).

[19] N. Shibata and D. Yoshioka, Ground-State Phase Diagram of 2D Electrons in a High Landau Level: A Density-Matrix Renormalization Group Study, Phys. Rev. Lett. 86, 5755 (2001).

[20] A. E. Feiguin, E. Rezayi, C. Nayak, and S. Das Sarma, Density Matrix Renormalization Group Study of Incompressible Fractional Quantum Hall States, Phys. Rev. Lett. 100, 166803 (2008).

[21] T. Xiang, Density-Matrix Renormalization-Group Method in Momentum Space, Phys. Rev. B 53, R10445 (1996).

[22] S. R. White, Density Matrix Formulation for Quantum Renormalization Groups, Phys. Rev. Lett. 69, 2863 (1992).

[23] J. Zhao, D. N. Sheng, and F. D. M. Haldane, Fractional Quantum Hall States at 1/3 and 5/2 Filling: DensityMatrix Renormalization Group Calculations, Phys. Rev. B 83, 195135 (2011).
[24] We thank A. H. MacDonald for pointing out this subtlety on the measurement of the Dipole moment for this state.

[25] D. J. Thouless, Quantization of Particle Transport, Phys. Rev. B 27, 6083 (1983).

[26] R. D. King-Smith and D. Vanderbilt, Theory of Polarization of Crystalline Solids, Phys. Rev. B 47, 1651(R) (1993).

[27] L. A. Falkovskii, Quasiclassical Quantization of Electrons and Holes in Bismuth in a Magnetic Field, J. Exp. Theor. Phys. 49, 609 (1965); A. Yu. Ozerin and L. A. Falkovsky, Berry Phase, Semiclassical Quantization, and Landau Levels, Phys. Rev. B 85, 205143 (2012).

[28] L. Onsager, Interpretation of the de Haas-van Alphen Effect, Philos. Mag. Ser. 5 43, 1006 (1952).

[29] C. Kittel, Introduction to Solid State (John Wiley \& Sons, New York, 1966).

[30] J. Liu, W. Duan, and L. Fu, Two Types of Surface States in Topological Crystalline Insulators, Phys. Rev. B 88, 241303(R) (2013).

[31] M. Serbyn and L. Fu, Symmetry Breaking and Landau Quantization in Topological Crystalline Insulators, Phys. Rev. B 90, 035402 (2014).

[32] I. Sodemann and L. Fu, Quantum Nonlinear Hall Effect Induced by Berry Curvature Dipole in Time-Reversal Invariant Materials, Phys. Rev. Lett. 115, 216806 (2015).

[33] The zeroth Landau level does not carry a dipole even in the presence of the tilt because the Hamiltonian retains an intravalley particle-hole times inversion symmetry, which forbids a dipole moment for the zeroth level. However, all other Landau levels will carry a dipole.

[34] D-W. Wang, E. Demler, and S. Das Sarma, Spontaneous Symmetry Breaking and Exotic Quantum Orders in Integer Quantum Hall Systems under a Tilted Magnetic Field, Phys. Rev. B 68, 165303 (2003).

[35] Y. Barlas, R. Cote, K. Nomura, and A. H. MacDonald, Intra-Landau-Level Cyclotron Resonance in Bilayer Graphene, Phys. Rev. Lett. 101, 097601 (2008).

[36] A. Zangwill, Modern Electrodynamics (Cambridge University Press, Cambridge, England, 2013).

[37] The stiffness cost is scale invariant and hence indifferent to the size of the skyrmion.

[38] The energetics can be captured by a nonlinear sigma model $[13,18]$, but using long-wavelength estimates for nonlinear sigma model parameters can lead to a substantial underestimation of the size of the skrymions. This is a known pitfall of this type of estimate for small skyrmions [39].

[39] M. Abolfath, J. J. Palacios, H. A. Fertig, S. M. Girvin, and A. H. MacDonald, Critical Comparison of Classical Field Theory and Microscopic Wave Functions for Skyrmions in Quantum Hall Ferromagnets, Phys. Rev. B 56, 6795 (1997).

[40] Quasihole and quasielectron excitations are related by particle-hole symmetry.

[41] D. Yoshioka, Ground State of the Two-Dimensional Charged Particles in a Strong Magnetic Field, and the Fractional Quantum Hall Effect, Phys. Rev. B 29, 6833 (1984).

[42] The method selects a single sector even in this case, in which there are SU(2)-related degenerate copies. 